\title{
Prevalence and Characterization of Mild Cognitive Impairment in Retired National Football League Players
}

\author{
Christopher Randolph, ${ }^{1}$ Stella Karantzoulis, ${ }^{2}$ AND Kevin Guskiewicz ${ }^{3,4,5}$ \\ ${ }^{1}$ Department of Neurology, Loyola University Medical Center, Maywood, Illinois \\ ${ }^{2}$ Department of Neurology, New York University Langone Medical Center, New York, New York \\ ${ }^{3}$ Department of Exercise and Sport Science, University of North Carolina at Chapel Hill, Chapel Hill, North Carolina \\ ${ }^{4}$ Department of Physical Medicine and Rehabilitation, University of North Carolina at Chapel Hill, Chapel Hill, North Carolina \\ ${ }^{5}$ Center for the Study of Retired Athletes, University of North Carolina at Chapel Hill, Chapel Hill, North Carolina
}

(Received March 22, 2013; Final Revision June 18, 2013; AcCePTEd June 18, 2013)

\begin{abstract}
It has been hypothesized that exposure to repetitive head trauma from contact sports over a long-playing career may eventuate in an increased risk of late-life cognitive impairment. There are currently two competing hypotheses about the possible mechanism underlying such impairment. One is the presence of a unique neurodegenerative disorder known as "chronic traumatic encephalopathy" (CTE). The other is diminished cerebral reserve leading to the earlier clinical expression of age-related neurodegenerative diseases such as mild cognitive impairment (MCI) and Alzheimer's disease (AD). The present study examined informant AD8 inventory data in a sample of 513 retired National Football League (NFL) players. These data were indicative of possible cognitive impairment in $35.1 \%$ of this relatively young sample. A comparison of neurocognitive profiles in a subsample of this group to a clinical sample of patients with a diagnosis of MCI due to AD revealed a highly similar profile of impairments. Overall, the data suggest that there may be an increased prevalence of late-life cognitive impairment in retired NFL players, which may reflect diminished cerebral reserve. The findings are considered preliminary, but emphasize the need for larger, controlled studies on this issue. (JINS, 2013, 19, 1-8)
\end{abstract}

Keywords: Concussion, Football, Sports, CTE, MCI

\section{INTRODUCTION}

There has been widespread media coverage and speculation regarding the late-life or post-retirement risks of cognitive impairment in athletes who engaged in sports involving repetitive head trauma. In contrast, there has been very little in the way of peer-reviewed scientific literature involving data that suggests any such risk. In 2005, we (Guskiewicz, et al., 2005) reported the results of a survey of retired NFL players that suggested a possible association between the number of concussions incurred during a player's career, and the risk of late-life cognitive impairment. In that study, an initial general health survey was sent to all 3683 living members of the retired players association; this survey included questions regarding concussion history. Of those, 2552 surveys were returned $(69 \%)$, and of that group, $61 \%$ of players reported sustaining at least one concussion, and $24 \%$ reported sustaining three or more concussions during their professional playing career.

Correspondence and reprint requests to: Christopher Randolph, 1 East Erie, Suite 353, Chicago, IL 60611. E-mail: crandol@lumc.edu
A follow-up survey focused on memory complaints was sent to all respondents who were over the age of 50 (1754), and this survey was returned by 758 retirees (43\%).

This survey included reporting of the clinical diagnosis of mild cognitive impairment (MCI) and Alzheimer's disease (AD), along with subjective complaints of memory impairment. Retired players with a history of three or more concussions had a five-fold increase in diagnosed MCI, and a three-fold increase in subjective memory problems compared to players with no history of concussion. Only 33 retired players $(1.3 \%)$ reported having been diagnosed by a physician as having $\mathrm{AD}$, and there was no apparent effect of concussion history on risk of $\mathrm{AD}$ diagnosis. Although the overall incidence of $\mathrm{AD}$ was low, this was a relatively young sample, and the incidence was higher than would be expected for the general population (age-adjusted prevalence ratio $=$ 1.37). This study was the first attempt to systematically explore late-life cognitive impairments in retired NFL players, and the results suggested the possibility of an increased incidence of late-life cognitive impairment, including $\mathrm{MCI}$ and $\mathrm{AD}$, in comparison to the general population. 
This study had limitations, however, and the most serious of these was that it was based upon data derived from general health questionnaires completed by the former player and closest relative. Despite reasonably good response rates for a survey $(69 \%, 43 \%)$, there is always a risk of ascertainment bias, and this could have resulted in an overestimation of the incidence of MCI and AD. Some additional support for these findings was recently supplied by a study of cause of death in retired NFL players (Lehman, Hein, Baron, \& Gersic, 2012). This study found elevated rates of death due to both AD and amyotrophic lateral sclerosis (ALS), despite a significantly lower overall mortality rate for retirees compared to the general population, matched for age, gender, and race. This study used a different database (pension fund) for NFL retirees than the study by Guskiewicz et al., and there was no associated ascertainment bias, providing a relatively independent validation of the earlier survey data. Nevertheless, the number of deaths was very low overall (two deaths attributed to $\mathrm{AD}$ and six deaths attributed to ALS, with AD as an associated diagnosis in an additional five cases, out of 334 total deaths). The much lower overall mortality rate for retirees $(\sim 50 \%$ of expected all-cause mortality) could also have resulted in disproportionate rates of deaths due to neurodegenerative causes in that population relative the general population. This suggests the need for continued study before reaching any conclusions about latelife risks associated with a long career in contact sports. Nevertheless, there are currently two competing hypotheses about potential pathophysiological mechanisms underlying such risks: chronic traumatic encephalopathy and diminished cerebral reserve leading to the earlier clinical expression of late-life neurodegenerative diseases.

\section{Chronic Traumatic Encephalopathy}

Chronic traumatic encephalopathy (CTE) is an entity that has been characterized by several different descriptions with respect to clinical and pathological criteria over the years. As described by recent publications, it is a progressive neurodegenerative disorder characterized by a variety of neuropsychiatric symptoms, although there remains a limited degree of clinical-pathological data upon which to base such a hypothesis. The term itself seems to date back at least to the 1960s, and was apparently used synonymously with dementia pugilistica. Dementia pugilistica is also a poorly defined syndrome, used in case-based descriptions of various neurological symptoms in boxers, with most of the reports dating back to the first half of the 20th century. Many of these cases may have been due to Alzheimer's disease or other neurodegenerative diseases, and the literature does not provide evidence of a unique disorder with clear clinical-pathological correlation. There also appears to have been some debate about whether CTE was progressive, or reflected a static encephalopathy. In a 1966 study, Miller pointed out the lack of any evidence suggesting that a neurodegenerative disorder could be triggered by head trauma, and with respect to CTE, stated "there is no firm evidence that dementia progresses once successive injuries have ceased" (Miller, 1966).
The term CTE seems to have fallen out of use for the most part after that point in time. In 1973, Corsellis and colleagues published a study on "punch drunk syndrome" in the context of a neuropathological study of the brains of 15 former boxers (Corsellis, Bruton, \& Freeman-Browne, 1973). These authors acknowledged the controversy about the syndrome, and the disparate clinical and neuropathological findings in the literature. They also acknowledged the inherent ascertainment bias in the fact that those boxers studied were more likely to come to autopsy because of the fact that they were institutionalized before their death. There were no neuropathological findings that were common to all 15 cases, but the most common findings were neurofibrillary tangles in the cortex, loss of pigmented cells in the substantia nigra, cavum septum pellucida, and cerebellar scarring. Senile plaques were observed in only 4 of 15 cases.

In what remains perhaps the best-controlled study of neuropathological findings in boxers to date, Roberts et al. re-examined 14 of the 15 brains from the Corsellis study, added 6 new cases of boxers, and compared these to 20 cases of Alzheimer's disease (AD) and 20 age-matched controls (Roberts, Allsop, \& Bruton, 1990). They used immunocytochemical methods for identifying amyloid plaques (in contrast to the silver staining done by Corsellis et al.). Their conclusion was that "The molecular markers present in the plaques and tangles of dementia pugilistica are the same as those in AD. Similarities in the clinical symptoms, distribution of pathology and neurochemical deficits also exist" (p. 373). Their essential conclusion was that dementia pugilistica and AD shared a common pathogenesis.

The term CTE was resurrected by Omalu et al. in two studies reporting neuropathological findings from two retired NFL players (Omalu et al., 2006, 2005). The first case was a 50-yearold man who died from a myocardial infarction. There were no documented premortem psychiatric or neurological diagnoses, or documented evidence of cognitive decline. The authors reported that telephone interviews with his family revealed evidence of a "dysthymic disorder." Pathological findings were diffuse amyloid plaques in the cortex and sparse cortical neuropil threads and neurofibrillary tangles (NFTs), without any subcortical evidence of these pathologies. The second case was a 45-year-old man who committed suicide by consuming antifreeze. He had a history of multiple psychiatric hospitalizations and prior suicide attempts, with a psychiatric diagnosis of major depression. Pathological findings were an absence of amyloid plaques, with diffuse cortical and subcortical NFTs and neuropil threads.

These studies were followed by a publication by McKee and colleagues in 2009, reporting on three additional cases: a retired NFL player who died from an accidental cause at age 45 , a retired boxer with a history of progressive dementia who died at age 80 , and a retired boxer with a history of progressive dementia who died at age 73 (McKee et al., 2009). The pathological findings of these three cases were also markedly disparate, with the only commonality being the presence of cortical NFTs and neuropils, although the distribution and density of these varied from case to case. 
The retired boxers who both had a history of progressive dementia also had evidence of gross atrophy and neuronal loss, with amyloid plaques evident in one case.

In more recent discussions of CTE, the focus has shifted to tau as the diagnostic hallmark. McKee et al. recently reported that they had identified evidence of CTE in $80 \%$ of subjects (age 17-98 years) with some history of repetitive head trauma. CTE was identified in 33/34 retired NFL players who constituted part of this sample. The authors proposed a staging system for CTE severity, and the only requirement for satisfying a neuropathological diagnosis of CTE was the presence of tau-positive astrocytic tangles and NFTs (McKee et al., 2013). Because NFTs are present in a variety of neurodegenerative disorders and are also evident in most normal older individuals, this classification criterion deserves further scrutiny.

As an example, NFTs (Braak stage 1 or higher) were identified in nearly all (97\%) of cognitively normal older individuals in a large prospective study, and $37 \%$ met criteria for a diagnosis of $\mathrm{AD}$ at an intermediate or high likelihood (Bennett et al., 2006). Although McKee et al. attempted to define a staging system for CTE and make some distinctions between AD and CTE pathologies, there remains substantial pathological overlap and a lack of clear clinical-pathological correlation. Even if repetitive head trauma led to abnormal phosphorylation of tau, it is unclear that this would have any direct behavioral effects. Without appropriate controls, therefore, it is impossible to determine whether these neuropathological findings in athletes exposed to repetitive head trauma are atypical, or whether there are any behavioral consequences thereof. Although there are currently no defined clinical diagnostic criteria for CTE, there have been some attempts to describe a clinical syndrome on the basis of retrospective review of pre-mortem information from selected cases. McKee and colleagues, for example, reported that, "CTE is clinically associated with symptoms of irritability, impulsivity, aggression, depression, short-term memory loss and heightened suicidality that usually begins 8-10 years after experiencing repetitive mild traumatic brain injury."(McKee et al., 2013) (p. 2). The claim of heightened suicidality is particularly interesting given the fact that retired NFL players have a significantly lower risk of suicide (less than half of the expected rate) than the general population of men their age in the United States (Baron, Hein, Lehman, \& Gersic, 2012).

\section{Diminished Cerebral Reserve}

In a review of the risks associated with sport-related concussion, Randolph and Kirkwood postulated that a long history of repetitive head trauma in contact sports might eventuate in diminished cerebral reserve, leading to the earlier expression of age-related neurodegenerative diseases like AD and Parkinson's disease (PD) (Randolph \& Kirkwood, 2009). They noted that a history of moderate-severe traumatic brain injury or stroke earlier in life has been demonstrated to result in the earlier clinical expression of neurodegenerative diseases, and suggested that a long history of repetitive minor head trauma might have a similar effect. They further hypothesized that concussion per se was unlikely to be as significant a factor as routine repetitive trauma, citing biomechanical studies that recorded extremely high rates of head impacts exceeding $10 \mathrm{~g}$ in high school and college football players, in some studies exceeding 1,000 such impacts on average per player-season (Duma et al., 2005; Mihalik, Bell, Marshall, \& Guskiewicz, 2007; Schnebel, Gwin, Anderson, \& Gatlin, 2007). It was additionally hypothesized that a history of multiple concussions might be a sign of diminishing reserve, as there is some evidence that concussion risk continues to rise with each additional concussive injury (Delaney, Lacroix, Leclerc, \& Johnston, 2000; Guskiewicz et al., 2003; Macciocchi, Barth, Littlefield, \& Cantu, 2001). This observation suggests some type of increasing sensitivity, possibly secondary to diminishing cerebral reserve.

\section{Hypothetical Predictions}

The hypothesis of diminished cerebral reserve would predict an earlier expression of age-related neurodegenerative diseases among individuals with a history of repetitive head trauma, but the clinical manifestation of those diseases (e.g., MCI, AD, PD) would not be expected to differ from individuals with the disease who lack such a history of trauma. In this hypothesis, the threshold for clinical expression is lowered, presumably due to neuronal cell loss, but any pathology that might be directly attributable to repetitive brain trauma (e.g., hyperphosphorylated tau) would not be expected to play a role in the neurodegenerative process or disease manifestation. Therefore, it would be predicted that MCI due to AD, for example, would be more prevalent in NFL retirees than in the general population of men in the same age range, but that the clinical profile of MCI would not differ between these groups.

The hypothesis that CTE is a distinct neurodegenerative disorder, on the other hand, would predict a unique clinical manifestation, related directly to neuropathological changes that are distinct from the pathologies associated with diseases such as MCI, AD, and PD. Although there remains a lack of any defined clinical criteria for diagnosing CTE, and no studies of neuropsychological functioning in individuals who have been reported to have neuropathological changes that are associated with CTE, it is reasonable to presume that CTE would be likely to produce a neurocognitive profile that differs from those observed in various other clinical conditions. The purpose of the present study was two-fold: (1) to follow up on the original NFL retiree study to further explore the prevalence of cognitive impairment in this sample, and; (2) to compare neuropsychological test data obtained on a sample of NFL retirees with diagnosed MCI to that of cognitively healthy controls and patients diagnosed with MCI due to AD who have no prior history of repetitive head trauma. The hypothesis of diminished cerebral reserve would predict a higher prevalence of cognitive impairment in this sample of our retired NFL players than would be expected in the general population, but that the neurocognitive profile of retired NFL players with MCI would be similar to that of a clinical sample 
of patients with MCI. Evidence of a different profile of impairments in the NFL sample, on the other hand, might be seen as support for the hypothesis of CTE underlying MCI in this sample.

\section{METHODS}

\section{Survey Data}

All data were obtained in accordance with human subject research guidelines at our respective institutions. In 2001, all retired NFL players affiliated with the NFL Player's Association $(N=3729)$ were mailed a general health survey. Of these surveys, 2536 were returned (68\% response rate). In 2008, an additional survey focusing on memory problems was sent out to all respondents who were over the age of 50 at the time $(N=908)$. This survey included the AD8 (Galvin et al., 2005), which is a screening tool that has been found to differentiate individuals who are cognitively intact from those with $\mathrm{MCI}$ and mild dementia. This is an eight-item questionnaire, designed primarily as an informant-based tool. Scores of 2 or higher are suggestive of clinically significant impairments of memory and cognition, although the validation of the instrument is still somewhat limited. These surveys were filled out by both the retirees and their spouses (where applicable). There were 633 surveys returned (70\% response rate). Of these, 513 had a spouse report. These 513 surveys were analyzed to explore rates of cognitive impairment in this sample.

\section{NFL MCI Sample and Controls}

As part of an industry-funded study of docosahexaenoic acid (DHA) in the treatment of MCI, an additional telephone screening was undertaken to identify retirees between the age of 50 and 75 who had probable MCI, using the modified; Telephone Interview for Cognitive Status (Welsh, Breitner, \& Magruder-Habib, 1993). A total of 41 retirees were identified as having probable MCI, and were recruited for this study, which was carried out by the Center for the Study of Retired Athletes in Chapel Hill, North Carolina. The neuropsychological testing done to characterize this sample at baseline included a short form of the Wechsler Adult Intelligence Test-III (WAIS-3) (Donnell et al., 2007) and the Repeatable Battery for the Assessment of Neuropsychological Status (RBANS) (Randolph, 1998).

To compare the NFL sample to a clinical sample, a sample of 81 patients with amnestic MCI was compiled from prospectively evaluated cases over 2 years from the pooled databases of two academically affiliated clinical centers (NYU and LUMC). All patients were diagnosed with MCI by a neuropsychologist according to the core clinical criteria of the revised NIA-AA guidelines (Albert et al., 2011) on the basis of: (1) observed decline by an informant in memory over the preceding year; (2) objective evidence of performance below expected premorbid levels in anterograde memory on standardized neuropsychological batteries in the judgment of the examining neuropsychologist; and (3) relative preservation of independence with respect to most activities of daily living. At each center, the typical standard of care also involved review of neuroimaging data and standard laboratories necessary to rule out metabolic or infectious etiologies for cognitive impairment, although those data were not collated for reporting here. This methodology differs somewhat from the clinical diagnostic approach used in some research samples, as informant-based observation was required (as opposed to relying upon subject self-report as equivalent), and there was no defined cutoff score for establishing "impairment." None of these patients had a history of involvement in professional contact sports. Finally, a sample of 41 cognitively normal subjects was extracted from the RBANS standardization database to match the NFL sample on the basis of age, education, and gender

\section{RESULTS}

\section{Survey Data}

Spousal AD8 data were analyzed for the 513 surveys that contained this information. The mean age of the retirees in this sample was $64.2(S D=5.5)$. In this sample, $35.1 \%$ of retirees were assigned a score of 2 or higher on the AD8, suggesting the presence of clinically significant cognitive impairment. The breakdown of AD8 scores is depicted in Figure 1.

\section{MCI in Retired NFL Players}

The sample of retirees was matched to a healthy control (HC) group extracted from the RBANS standardization database on the basis of age, gender, and education, and as a result the groups did not differ on any of these demographic variables (see Table 1). Despite having a fairly high mean Full-Scale IQ on the WAIS-3 short form (110.8), the RBANS Total Scale score mean for the NFL sample was significantly below that of the HC sample, $F=10.4 ; p=.002$; Cohen's $d=1.5$ (effect size 0.6). This would indicate the likelihood of

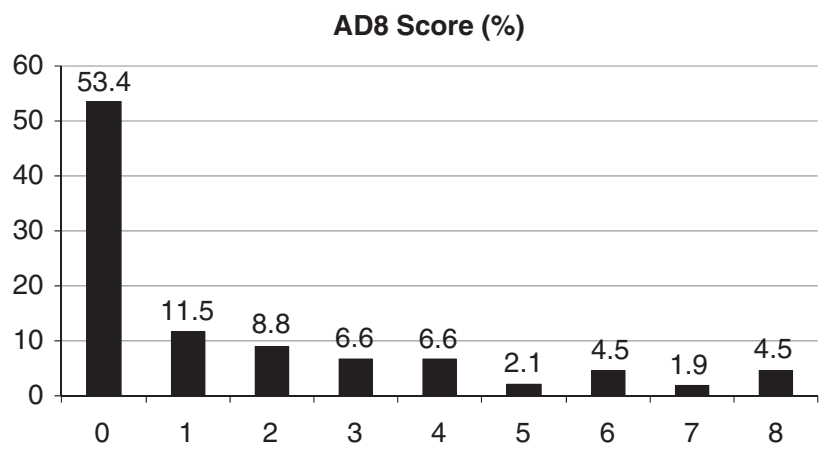

Fig. 1. Breakdown of spousal-provided AD8 scores for the 513 National Football League (NFL) retirees that returned surveys. Scores of 2 or higher are interpreted as indicative of clinically significant impairment of cognition; $35.1 \%$ of the total sample fell into this category. 
Table 1. Descriptive statistics

\begin{tabular}{lccr}
\hline \hline & MCI $(n=81)$ & NFL $(n=41)$ & HC $(n=41)$ \\
\hline Age & $77.3(6.8)$ & $64.2(5.5)$ & $64.0(5.8)$ \\
Education & $15.4(2.7)$ & $16.3(1.3)$ & $15.6(3.4)$ \\
Sex (M:F) & $47: 34$ & $41: 0$ & $41: 0$ \\
Full-Scale IQ & $106.5(13.6)$ & $110.8(15.6)$ & N/A \\
RBANS Immediate Memory & $83.0(14.1)$ & $89.4(14.5)$ & $101.2(12.7)$ \\
RBANS Visuospatial/Constructional & $87.8(17.2)$ & $94.9(12.5)$ & $102.3(15.0)$ \\
RBANS Language & $89.7(11.6)$ & $96.8(9.4)$ & $97.4(10.1)$ \\
RBANS Attention & $96.4(14.6)$ & $103.0(14.9)$ & $102.7(14.6)$ \\
RBANS Delayed Memory & $73.5(16.5)$ & $87.6(17.5)$ & $101.7(14.5)$ \\
RBANS Total Scale Score & $81.7(11.1)$ & $92.0(12.0)$ & $100.6(13.8)$ \\
AD8 & N/A & $2.9(3.2)$ & N/A \\
\hline \hline
\end{tabular}

Note. All data (apart from sex) are means $(S D) . \mathrm{MCI}=$ clinical sample of $\mathrm{MCI}$ due to Alzheimer's disease; $\mathrm{NFL}=$ retirees with mild cognitive impairment; $\mathrm{HC}=$ healthy controls; $\mathrm{N} / \mathrm{A}=$ not available; RBANS $=$ Repeatable Battery for the Assessment of Neuropsychological Status.

acquired cognitive impairment. The mean spousal AD8 score for this NFL sample was 2.9 (Table 1). A mean score of 2.9 on the AD8 was also reported by Galvin and colleagues (Galvin et al., 2005) for a sample of clinically defined patients with MCI, further suggesting that this NFL sample was characterized by mild, but clinically significant impairment of cognition.

In comparing the NFL retirees with MCI to the clinical sample of patients with MCI, the NFL retiree sample entirely male, of course, and was significantly younger $(F=113.4$; $p<.0001)$. This was likely due in part to an established upper age cutoff of 75 for recruitment of the NFL retiree sample. The NFL retiree sample also had a slightly higher WAIS-3 short form FSIQ (110.8 vs. 106.5), but this was not statistically significant. To compare RBANS profiles, the five domain-based index scores for the RBANS were entered into a multivariate analysis of variance comparing these two groups. There was an overall effect of group $(F=22.4$; $p<.0001)$, and of domain $(F=27.5, p<.0001)$, but there was no group by domain interaction $(F=1.98$, NS). This indicates that the overall profile of performance across domains was similar for the two groups (see Figure 2). A similar analysis was conducted by gender within the clinical sample, to ensure that there were no gender-based differences that might have obscured such an interaction. There was no effect of gender or gender by domain interaction, indicating that the male and female subjects within the clinical MCI sample had a similar overall level and pattern of impairment.

Cognitive impairment in the retired NFL sample was also explored as a function of years of professional play. The mean number of years played in the NFL for this sample was $7.5(S D=3.4)$. There was no correlation between years played and RBANS Total Scale score $(r=.016, \mathrm{NS})$.

\section{DISCUSSION}

The late-life effects of repetitive head trauma incurred in contact sports remain largely unknown, although they have garnered a great deal of recent attention. To date, there has not yet been a well-controlled study exploring the prevalence of long-term cognitive or behavioral impairments in any such sport, including boxing or American football. In addition, the neuropathological correlates of this type of repetitive head trauma also remain unclear. No prospective study has explored the prevalence of any particular neuropathological finding (NFTs, amyloid plaques, cerebellar scarring) in these athletes compared to non-athlete controls. In the absence of prospective studies, it is unclear if any particular pathology can be solely attributed to repetitive head trauma, and whether it plays a role in the genesis of any behavioral or cognitive changes.

Over the past decade, there has been well-publicized speculation about the causal relationships linking participation in contact sports and the presence of CTE. The 2013 consensus statement by the International Consensus Group

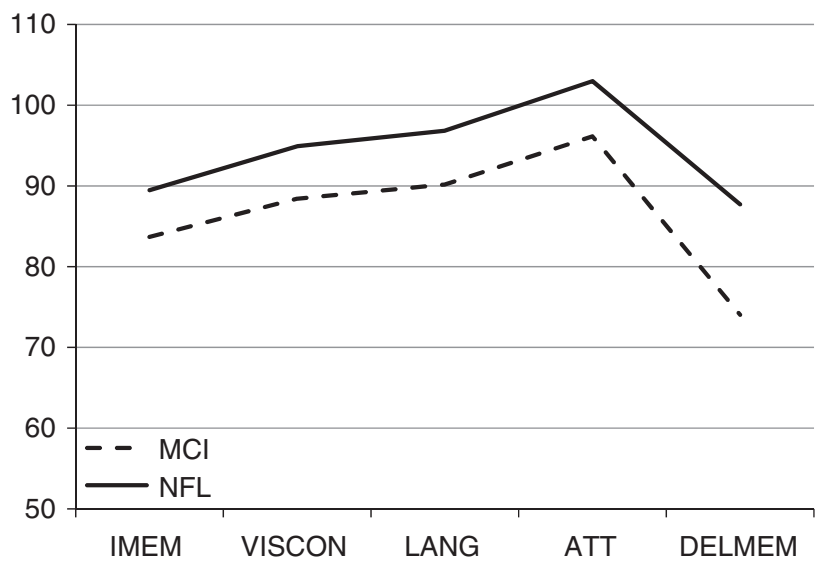

Fig. 2. Mean index scores on each of the five Repeatable Battery for the Assessment of Neuropsychological Status (RBANS) domains for the sample of National Football League (NFL) retirees with cognitive impairment $(N=41)$ and clinically diagnosed patients with MCI $(N=81)$. IMEM, Immediate Memory; VISCON, Visuospatial/Constructional; LANG, Language; ATT, Attention; DELMEM, Delayed Memory. 
on Concussion in Sport concluded, however, that there is insufficient data to support the association, stating that "It was agreed that CTE represents a distinct tauopathy with an unknown incidence in athletic populations. It was further agreed that a cause and effect relationship has not yet been demonstrated between CTE and concussions or exposure to contact sports." (McCrory et al., 2013). Whereas early researchers in this area seemed to have considered CTE to be a static encephalopathy (Miller, 1966), more recently it has been proposed that CTE is a progressive tauopathy characterized by cognitive and behavioral changes, suicidality, and progressive dementia (McKee et al., 2013). Alternatively, it has been proposed that repetitive head trauma from contact sports over a long career may result in diminished cerebral reserve, leading to the earlier clinical expression of neurodegenerative disorders such as MCI/AD (Randolph \& Kirkwood, 2009).

The present study explored the prevalence of spousalreported cognitive impairment in an extensive health questionnaire of retired NFL players. Just over 35\% of the sample was reported by their spouse to be exhibiting evidence of clinically significant impairment of cognition. This is a surprisingly high percentage, particularly given the relatively young mean age of the sample (64.2). Most epidemiological studies of MCI are done in older age groups, but prevalence rates are typically much lower, and usually under $5 \%$ for men under the age of 75 (Roberts et al., 2012; Tervo et al., 2004). This is certainly suggestive of the possibility that there is an increased incidence of late-life cognitive impairment in retired NFL players.

In examining the profile of neurocognitive impairment in the retired NFL players who were recruited for the MCI study, there was no difference between this group and the sample of patients carrying a clinical diagnosis of MCI in RBANS domain score profile. The NFL sample was significantly younger as a whole, but this may have been due in part to the age restriction in recruitment. The NFL sample also had a milder level of impairment overall, which is not unexpected since they were actively recruited as opposed to the clinical sample, which consisted of patients who were referred for neuropsychological evaluation of clinically evident cognitive decline. The fact that the pattern of performance across the RBANS domain scores was essentially identical across groups suggests that a common pathophysiology underlies the cognitive impairments exhibited by each group. The RBANS domain scores have been demonstrated to differ in profile across dementia of different etiologies (Beatty et al., 2003; Randolph, Tierney, Mohr, \& Chase, 1998), included markedly different profiles for AD and well-defined tauopathy, progressive supranuclear palsy (Randolph, Gold, \& Morimoto, 2012). The lack of any such difference here would tend to support the hypothesis of diminished cerebral reserve rather than the presence of CTE. These results are consistent with another recent study of NFL retirees (Hart et al., 2013). This study actively recruited retirees with cognitive and neurobehavioral symptoms, with a total sample size of 34. They concluded that rates of MCI and depression were slightly higher than would be expected for the population but that "none of the retired players fit the reported profile for CTE at the time of examination" (p. 331).

Although there was no relationship between the number of years played and degree of cognitive impairment in the present study, that finding has little bearing upon the hypothesis of diminished cerebral reserve. This sample was specifically recruited for the probable presence of mild cognitive impairment. Such a correlation would be more likely to emerge in a random selection of NFL retirees, rather than a subgroup selected for a specific degree of cognitive impairment (or an absence thereof), which constrains the range of impairment. In addition, cumulative trauma varies by position (Broglio et al., 2011), and we lacked historical playing position data that would be necessary to attempt to calculate a cumulative trauma exposure score. Finally, it is generally believed that diminished cerebral reserve is likely to interact with other risk factors (e.g., ApoE genotype, vascular risk factors) in contributing the emergence of clinically evident cognitive decline. A much larger sample of randomly selected retirees with more detailed information on cumulative trauma exposure and other risk factors would be necessary to parse out the potential effects of cumulative head trauma from football in contributing to the onset of late-life cognitive decline.

There are several important limitations to this study. The AD8 data were based upon information obtained from a survey, and despite the relatively good response rate, there may have been an ascertainment bias in terms of which players returned the survey. In addition, there were no controls in this section of the study, and the rate of probable cognitive impairment had to be compared to published prevalence rates. The validation of the AD8 is still somewhat limited, and the false-positive rates in the healthy population are not well-established.

In comparing the neuropsychological profiles of the NFL players with mild cognitive impairment to the clinical sample with MCI due to AD, we were limited to the WAIS-III SFIQ and the RBANS, because these were the only neuropsychological measures given to the NFL sample that were also available for the MCI clinical sample. The inclusion of "executive" measures and of a neuropsychiatric inventory across all subjects would have allowed us to further characterize each sample, and to rule out any other differences in clinical phenomenology. We acknowledge this as a limitation of our study. The NFL retirees were also recruited on the basis of some cognitive impairment in the absence of obvious dementia, and that may have resulted in an ascertainment bias, resulting in the identification of individuals who were more clinically similar to patients with MCI due to $\mathrm{AD}$ at the expense of recruiting individuals who may have had a different profile of cognitive or behavioral impairments.

Although these data suggest that there is a higher prevalence of cognitive impairment in retired NFL players than expected by population estimates, and that MCI in retired NFL players appears to be very similar in neurocognitive profile to MCI due to AD in a clinical sample, these conclusions remain very tentative. The media attention to this issue continues to far outweigh any meaningful results 
from sound experimental science, and a definitive epidemiological study still has yet to be done. The most useful approach to this problem would be to conduct a truly random sampling of the at-risk population (e.g., retired NFL players) of a sufficient sample size that could be compared to an appropriate control group to determine whether or not there is an increased prevalence of cognitive impairment in the at-risk group. If this is confirmed, a prospective controlled study of the characteristics, natural history, genotype, and underlying neuropathophysiology would be enabled, and this would result in definitive clinicopathological information. Until such a study is conducted, a conservative approach to interpreting pathological findings of uncertain clinical significance in case reports is warranted.

\section{ACKNOWLEDGMENTS}

This study was funded in part by Martek Biosciences Corporation. We thank Candice Goerger, Amy Matthews, Karla Thompson, and Daniel Kaufer for their contributions. Dr. Randolph is the author of the RBANS, and receives royalties from sales of this test. The authors have no other conflicts to disclose

\section{REFERENCES}

Albert, M.S., DeKosky, S.T., Dickson, D., Dubois, B., Feldman, H.H., Fox, N.C., ... Phelps, C.H. (2011). The diagnosis of mild cognitive impairment due to Alzheimer's disease: Recommendations from the National Institute on Aging-Alzheimer's Association workgroups on diagnostic guidelines for Alzheimer's disease. Alzheimers and Dementia, 7, 270-279.

Baron, S.L., Hein, M.J., Lehman, E., \& Gersic, C.M. (2012). Body mass index, playing position, race, and the cardiovascular mortality of retired professional football players. American Journal of Cardiology, 109, 889-896.

Beatty, W.W., Ryder, K.A., Gontkovsky, S.T., Scott, J.G., McSwan, K.L., \& Bharucha, K.J. (2003). Analyzing the subcortical dementia syndrome of Parkinson's disease using the RBANS. Archives of Clinical Neuropsychology, 18, 509-520.

Bennett, D.A., Schneider, J.A., Arvanitakis, Z., Kelly, J.F., Aggarwal, N.T., Shah, R.C., \& Wilson, R.S. (2006). Neuropathology of older persons without cognitive impairment from two community-based studies. Neurology, 66, 1837-1844.

Broglio, S.P., Eckner, J.T., Martini, D., Sosnoff, J.J., Kutcher, J.S., \& Randolph, C. (2011). Cumulative head impact burden in high school football. Journal of Neurotrauma, 28, 2069-2078.

Corsellis, J.A., Bruton, C.J., \& Freeman-Browne, D. (1973). The aftermath of boxing. Psychological Medicine, 3, 270-303.

Delaney, J.S., Lacroix, V.J., Leclerc, S., \& Johnston, K.M. (2000). Concussions during the 1997 Canadian Football League season. Clinical Journal of Sports Medicine, 10, 9-14.

Donnell, A.J., Pliskin, N., Holdnack, J., Axelrod, B., \& Randolph, C. (2007). Rapidly-administered short forms of the Wechsler Adult Intelligence Scale-3rd edition. Archives of Clinical Neuropsychology, 22, 917-924.

Duma, S.M., Manoogian, S.J., Bussone, W.R., Brolinson, P.G., Goforth, M.W., Donnenwerth, J.J., Greenwald, R.M., Chu, J.J., \& Crisco, J.J. (2005). Analysis of real-time head accelerations in collegiate football players. Clinical Journal of Sports Medicine, $15,3-8$.
Galvin, J.E., Roe, C.M., Powlishta, K.K., Coats, M.A., Muich, S.J., Grant, E., Miller, J.P., Storandt, M., \& Morris, J.C. (2005). The AD8: A brief informant interview to detect dementia. Neurology, $65,559-564$.

Guskiewicz, K.M., Marshall, S.W., Bailes, J., McCrea, M., Cantu, R.C., Randolph, C., \& Jordan, B.D. (2005). Association between recurrent concussion and late-life cognitive impairment in retired professional football players. Neurosurgery, 57, 719-726; discussion 719-726.

Guskiewicz, K.M., McCrea, M., Marshall, S.W., Cantu, R.C., Randolph, C., Barr, W.B., Onante, J.A., \& Kelly, J.P. (2003). Cumulative effects associated with recurrent concussion in collegiate football players: The NCAA concussion study. Journal of the American Medical Association, 290, 2549-2555.

Hart, J. Jr, Kraut, M.A., Womack, K.B., Strain, J., Didehbani, N., Bartz, E., Conover, H., Mansinghani, S., Lu, H., \& Cullum, C.M. (2013). Neuroimaging of cognitive dysfunction and depression in aging retired National Football League players: A cross-sectional study. JAMA Neurology, 70, 326-335.

Lehman, E.J., Hein, M.J., Baron, S.L., \& Gersic, C.M. (2012). Neurodegenerative causes of death among retired National Football League players. Neurology, 79, 1970-1974.

Macciocchi, S.N., Barth, J.T., Littlefield, L.M., \& Cantu, R. (2001). Multiple concussions and neuropsychological functioning in collegiate football players. Journal of Athletic Training, 303-306.

McCrory, P., Meeuwisse, W.H., Aubry, M., Cantu, B., Dvorak, J., Echemendia, R.J., ... Turner, M. (2013). Consensus statement on concussion in sport: the 4th International Conference on Concussion in Sport held in Zurich, November 2012. British Journal of Sports Medicine, 47, 250-258.

McKee, A.C., Cantu, R.C., Nowinski, C.J., Hedley-Whyte, E.T., Gavett, B.E., Budson, A.E., ... Stern, R.A. (2009). Chronic traumatic encephalopathy in athletes: Progressive tauopathy after repetitive head injury. Journal of Neuropathology and Experimental Neurology, 68, 709-735.

McKee, A.C., Stein, T.D., Nowinski, C.J., Stern, R.A., Daneshvar, D.H., Alvarez, V.E., ... Cantu, R.C. (2013). The spectrum of disease in chronic traumatic encephalopathy. Brain, 136(Pt 1), 43-64.

Mihalik, J.P., Bell, D.R., Marshall, S.W., \& Guskiewicz, K.M. (2007). Measurement of head impacts in collegiate football players: an investigation of positional and event-type differences. Neurosurgery, 61, 1229-1235; discussion 1235.

Miller, H. (1966). Mental after-effects of head injury. Proceedings of the Royal Society of Medicine, 59, 257-261.

Omalu, B.I., DeKosky, S.T., Hamilton, R.L., Minster, R.L., Kamboh, M.I., Shakir, A.M., \& Wecht, C.H. (2006). Chronic traumatic encephalopathy in a national football league player: part II. Neurosurgery, 59, 1086-1092; discussion 1092-1083.

Omalu, B.I., DeKosky, S.T., Minster, R.L., Kamboh, M.I., Hamilton, R.L., \& Wecht, C.H. (2005). Chronic traumatic encephalopathy in a National Football League player. Neurosurgery, 57, 128-134; discussion 128-134.

Randolph, C. (1998). Repeatable Battery for the Assessment of Neuropsychological Status (RBANS). San Antonio, TX: The Psychological Corporation.

Randolph, C., Gold, M., \& Morimoto, B. (2012). The Repeatable Battery for the Assessment of Neuropsychological Status (RBANS) in progressive supranuclear palsy: Baseline data from the Allon Therapeutics AL-108-231 multinational clinical trial. Alzheimer's \& Dementia: The Journal of the Alzheimer's Association, 8, 172. 
Randolph, C., \& Kirkwood, M.W. (2009). What are the real risks of sport-related concussion, and are they modifiable? Journal of the International Neuropsychological Society, 15, 512-520.

Randolph, C., Tierney, M.C., Mohr, E., \& Chase, T.N. (1998). The Repeatable Battery for the Assessment of Neuropsychological Status (RBANS): Preliminary clinical validity. Journal of Clinical and Experimental Neuropsychology, 20, 310-319.

Roberts, G.W., Allsop, D., \& Bruton, C. (1990). The occult aftermath of boxing. Journal of Neurology, Neurosurgery, and Psychiatry, 53, 373-378.

Roberts, R.O., Geda, Y.E., Knopman, D.S., Cha, R.H., Pankratz, V.S., Boeve, B.F., ... Petersen, R.C. (2012). The incidence of MCI differs by subtype and is higher in men: the Mayo Clinic Study of Aging. Neurology, 78, 342-351.
Schnebel, B., Gwin, J.T., Anderson, S., \& Gatlin, R. (2007). In vivo study of head impacts in football: a comparison of National Collegiate Athletic Association Division I versus high school impacts. Neurosurgery, 60, 490-495; discussion 495-496.

Tervo, S., Kivipelto, M., Hanninen, T., Vanhanen, M., Hallikainen, M., Mannermaa, A., \& Soininen, H. (2004). Incidence and risk factors for mild cognitive impairment: a population-based threeyear follow-up study of cognitively healthy elderly subjects. Dementia and Geriatric Cognitive Disorders, 17, 196-203.

Welsh, K., Breitner, J., \& Magruder-Habib, K. (1993). Detection of dementia in the elderly using Telephone Screening of Cognitive Status. Neuropsychiatry Neuropsychology and Behavioral Neurology, 6, 103-110. 